\title{
What Physical Processes Drive the Interstellar Medium in the Local Bubble?
}

\author{
D. Breitschwerdt • M.A. de Avillez $\cdot$ B. Fuchs $\cdot$ C. Dettbarn
}

Received: 28 March 2008 / Accepted: 30 July 2008 / Published online: 12 September 2008

(C) Springer Science+Business Media B.V. 2008

\begin{abstract}
Recent 3D high-resolution simulations of the interstellar medium in a star forming galaxy like the Milky Way show that supernova explosions are the main driver of the structure and evolution of the gas. Its physical state is largely controlled by turbulence due to the high Reynolds numbers of the average flows. For a constant supernova rate a dynamical equilibrium is established within $200 \mathrm{Myr}$ of simulation as a consequence of the setup of a galactic fountain. The resulting interstellar medium reveals a typical density/pressure pattern, i.e. distribution of so-called gas phases, on scales of 500-700 pc, with interstellar bubbles being a common phenomenon just like the Local Bubble and the Loop I superbubble, which are assumed to be interacting. However, modeling the Local Bubble is special, because it is driven by a moving group, passing through its volume, as it is inferred from the analysis of Hipparcos data. A detailed analysis reveals that between 14 and 19 supernovae have exploded during the last $15 \mathrm{Myr}$. The age of the Local Bubble is derived from comparison with HI and UV absorption line data to be $14.5 \pm_{0.4}^{0.7} \mathrm{Myr}$. We further predict the merging of the two bubbles in about $3 \mathrm{Myr}$ from now, when the interaction shell starts to fragment. The Local Cloud and its companion HI clouds are the consequence of a dynamical instability in the interaction shell between the Local and the Loop I bubble.
\end{abstract}

Keywords Interstellar matter $\cdot$ Superbubbles $\cdot$ Local bubble $\cdot$ Turbulence

D. Breitschwerdt ( $₫)$

Institut für Astronomie, University of Vienna, Türkenschanzstraße 17, 1180 Vienna, Austria

e-mail: breitschwerdt@astro.univie.ac.at

M.A. de Avillez

Department of Mathematics, University of Évora, R. Romão Ramalho 59, 7000 Évora, Portugal

B. Fuchs · C. Dettbarn

Astronomisches Rechen-Institut, ZAH Universität Heidelberg, Mönchhofstraße 12-14,

69120 Heidelberg, Germany 


\section{Introduction}

The interstellar medium (ISM) in galaxies like our Milky Way is continuously evolving by forming stars in dense and cool clouds, and by enriching the gas with heavy elements from stellar explosions thus closing a galactic matter cycle. It has been known for a long time that such feedback mechanisms regulate star formation. More debatable, and hence less accepted, was the proposed outflow of hot gas from the disk as a galactic fountain (Shapiro and Field 1976; Bregman 1980; Kahn 1981; de Avillez 2000; Breitschwerdt and Komossa 2000) or even as a wind (e.g. Mathews and Baker 1971; Breitschwerdt et al. 1991; Everett et al. 2008) driven by supernova heated plasma and cosmic rays. One reason for skepticism lay in the lack of direct observational evidence. Absorption line studies of the galactic halo (e.g. Savage and de Boer 1981) were overwhelmingly pointing to negative velocities and hence infalling gas. However, the existence of hot

gaseous X-ray halos observed with ROSAT, and now with Chandra and XMM-Newton, argue beyond doubt for outflow in star forming galaxies like NGC 891 (Tüllmann et al. 2006) sometimes labeled as a twin of our galaxy.

Why is the outflow from the disk into the halo so important for the evolution of the disk ISM? The simplest answer is: it acts as a pressure release valve. Without it, most of the disk volume, perhaps 50-70\% as envisaged by McKee and Ostriker (1977), would be occupied by the hot medium. The evolution of a superbubble like our Local Bubble (LB), in which the solar system is embedded, would be quite different in a high pressure, low density environment. However, the story to tell about the ISM in general and the local ISM (LISM) in particular is not quite as simple and more subtle. In the ISM the estimated Reynolds' numbers are quite high, about $10^{5}-10^{7}$ (Elmegreen and Scalo 2004), and the gas is highly compressible. Therefore its dynamical and thermal state is largely determined by turbulence, as was first pointed out by von Weizsäcker (1951), which can be both supersonic and superalfvénic. The implications for the evolution of the ISM, as we shall show in the following sections, are profound. Turbulence does not add only extra pressure to the medium, as it is often simplistically treated, but also transfers energy from larger to smaller scales, or vice versa (inverse cascade), and promotes efficient mixing of chemically enriched material allowing for the existence of gas in classical thermally unstable density-temperature regimes.

From all we know the LISM on scales of a few hundred parsecs is not vastly different from the general ISM. Star formation seems to be ongoing as can be inferred from the existence of several nearby superbubbles. The Loop I superbubble, with the North Polar Spur being its shock illuminated outer shell and also the largest coherent X-ray structure in the sky, is excited by the Sco Cen association (or one of its distinct subgroups). On the other hand, there is no young stellar cluster known inside the LB. However, a detailed investigation of Hipparcos and radial velocity data of the local stellar population revealed (Berghöfer and Breitschwerdt 2002; Fuchs et al. 2006) the existence of a moving group passing through the volume occupied by the LB during the last 15 million years and generating the LB by the successive explosions of about $20 \mathrm{SNe}$.

This review paper is organized as follows: In Sect. 2 the modeling of the general ISM and the LB are discussed, while in Sect. 3 the results of our models are compared with observations, notably in HI and UV. In Sect. 4 we summarize our results and present some conclusions.

\section{Modeling the ISM and the Local Bubble}

The limitations of analytical and semianalytical models of the state and evolution of the ISM are obvious. One is forced to make assumptions, e.g. of global pressure equilibrium, 
mass balance, etc. like in the so-called three phase model. Transitions from one phase to another are instantaneous and regulated by the temperature perturbations at constant pressure of the net heat loss function $\mathcal{L}=n^{2} \Lambda(T)-n \Gamma$, where $n, T, \Lambda, \Gamma$ are the number density, temperature, interstellar metallicity dependent cooling and heating functions, respectively. According to Field's (1965) criterion $\left(\frac{\partial \mathcal{L}}{\partial T}\right)_{P}>0$ guarantees stability of a gas phase. However, as we shall see, this is only true if the cooling time is less than the dynamical time scale. In particular turbulence can have a stabilizing effect on gas in the transition regimes.

Turbulent flows are fundamentally different in nature from laminar flows. Generally, turbulence is fed by the mean flow field via Reynolds stresses, which arise from the randomly fluctuating velocity components in a fluid. Much insight can be gained by looking at the evolution and distribution of vorticity $(\omega=\nabla \times \mathbf{u})$. In turbulence, the velocities are correlated over a characteristic distance, which can be described by so-called "eddies". The largest eddies contain the bulk of the kinetic energy and their characteristics are often depending directly on the nature of the source. Since vorticity can neither be created nor destroyed in the interior of a flow, but rather in boundary layers, its evolution is determined by diffusion and stretching of turbulent blobs. The latter leads to the break-up of eddies into smaller structures and a subsequent cascade of energy from the largest to the smallest scales, where viscous dissipation sets in. A simple manifestation of a turbulent flow is shear flow. In the ISM a lot of shear is introduced by interacting flows or the interaction of pressure driven flows with the ambient medium. Therefore a multitude of sources for turbulence exists: stellar (e.g. from $\mathrm{HII}$ regions, stellar winds, $\mathrm{SNe}$ ), differential galactic rotation, fluid instabilities (Kelvin-Helmholtz, Parker, magnetorotational instability, etc.), self-gravity, etc. An estimate of their relevance in the ISM has been carried out with the result that SNe dominate by at least a factor of a few (MacLow and Klessen 2004). Therefore it seems justified to start numerical investigations of the evolution of the ISM with SNe (and stellar winds) as the driving sources of mass, momentum and energy input. We note, however, that our simulations like those of other groups, suffer from a fundamental shortcoming: we cannot resolve the viscous scale, where energy dissipation occurs. This problem is well known in turbulence studies, and there exist basically two approaches. In direct numerical simulations (DNS), the maximum Reynolds number is limited to about $R e \sim 1000$, since in incompressible turbulence $R e \sim\left(L / l_{\text {box }}\right)^{4 / 3} N_{x}^{4 / 9}$, where $L, l_{\text {box }}$, and $N_{x}$ are the outer scale at which turbulence is fed in, the box size, and the number of data points required at any instant, respectively. For ISM simulations this is far below realistic Reynolds numbers. In connection with laboratory studies, large eddy simulations (LES) are carried out, in which only the largest scales are resolved, since they only require a tiny fraction of the computing time, and yet carry the most important properties of turbulence, like e.g. the energy flux cascading down to smaller scales. To cut off the flow at some wavenumber $k_{\max }$ can only be done at the price of supplying an empirically motivated closure model (from comparison to laboratory studies), which accounts for the hopefully correct energy drainage out of the turbulent cascade. In case of the ISM this is far from trivial and is the subject of ongoing research.

In the following we give a brief overview on modeling a SN driven ISM on large and small scales.

\subsection{A Supernova Driven ISM Model}

Before going into details, a few general remarks are appropriate. Modeling a whole galaxy with sufficiently high resolution is beyond the current limit of computing power. We have therefore chosen a representative patch of the ISM, large enough to cover all, even the largest, structures, and small enough to still retain high resolution locally. A common feature in our models is the inclusion of the complete large scale disk-halo-disk circulation 
flow, and, as a prerequisite of this, a sufficiently long evolution time of typically $400 \mathrm{Myr}$. Calculations have shown that simulations with dynamical time scales shorter than $200 \mathrm{Myr}$ still bear memory effects of the initial conditions, as the fountain return flow time is of about the same order. Another feature of the models to be described is that they contain the most important physical processes, but are not exhaustive in the sense, that we need to understand their physical implications first, before we add new physics to the model. In this "bottom-up" manner we have included so far: gravitational field of stars, adopted from Kuijken and Gilmore (1989) (but not self-gravity, which is currently tested), detailed interstellar heating and cooling processes assuming an optically thin gas in collisional ionization equilibrium (CIE, i.e. ionization rate by collisions between electrons and ions is balanced by radiative recombination rate) using the cooling functions of Sutherland and Dopita (1993) for $T \geq 10^{4} \mathrm{~K}$ and Dalgarno and McCray (1972) for $T<10^{4} \mathrm{~K}$, with a temperature cut-off at $10 \mathrm{~K}$, and uniform heating due to starlight varying with height $z$ (Wolfire et al. 1995).

The computational box was chosen to be $\Delta x \times \Delta y \times \Delta z=(1 \times 1 \times \pm 10)$ (in units of kpc) on a Cartesian grid, centred on the solar circle, and evolved on parallel processors with adaptive mesh refinement (AMR). The hydrodynamics was based on a Riemann solver scheme using the piecewise parabolic method with Lagrangian remap (PPMLR). For further details see de Avillez and Breitschwerdt (2004), and for the setup of the MHD runs de Avillez and Breitschwerdt (2005a). We have included SNe Types Ia, b, c and Type II with their appropriate scale heights taken from observation. After terminating their main sequence life time, half of these explosions occur in a clustered fashion, the others randomly in the field. As a criterion for stars to form we have applied a density/temperature threshold criterion of $n>10 \mathrm{~cm}^{-3}, T<100 \mathrm{~K}$ if a sufficient amount of mass is available to distribute the stars according to their initial mass function (IMF). Stars drift away from their parent association with a random velocity of $\sim 5 \mathrm{~km} / \mathrm{s}$.

\subsection{Results on the General ISM}

After having reached global dynamical equilibrium after about $200 \mathrm{Myr}$, the initial conditions for the setup have been completely erased. In Fig. 1 it can be seen that both the density and temperature distributions in the Galactic midplane span more than 5 orders of magnitude in values and that the morphology of the gas is very filamentary. It is interesting to note that the probability distribution functions (PDFs) in Fig. 7 of de Avillez and Breitschwerdt (2005a, 2005b) for density of the warm HI and the diffuse ionized gas (DIG) show variations of about 3-4 orders of magnitude at the level of 0.01, and that the PDFs represent a lognormal distribution. This is more or less exactly what has been found by Berkhuijsen and Fletcher (2008) in recent observations of a sample of pulsars by analyzing the dispersion and emission measures.

Textbook superbubbles are found nowhere, except if they are caught during their very early stages of evolution. Interestingly the pressure distribution (middle panel of Fig. 1) shows also large variations, not only locally, but also on larger scales. This means that the pressure in the ISM is certainly not uniform, as one of the key assumptions in the model of McKee and Ostriker (1977) requires. This may again be regarded as an effect of turbulence, since ram pressure dominates the flow between $10^{2}-10^{6} \mathrm{~K}$, as can be inferred from Fig. 2 (bottom). Finally, as a consequence of the existence of a large scale galactic fountain, the volume filling factors are also vastly different from equilibrium models. In our simulations (both HD and MHD) the filling factor of the hot gas is always less than 20\% (see Fig. 2 top). Another surprising result is that the gas is not distributed into distinct phases like in the classical model, but also exists in intermediate and thermally unstable temperature regimes. This is most certainly due to the stabilizing effect of turbulence on local condensation modes. 
Fig. 1 Two-dimensional cuts through the 3D data cube: number density $n$ (top), thermal pressure in units of Boltzmann's constant (cgs), $P / k$ (middle), and temperature, $T$ in K (bottom), distribution in the Galactic midplane plane for a Galactic supernova rate $\left(\sigma / \sigma_{G a l}=1\right)$
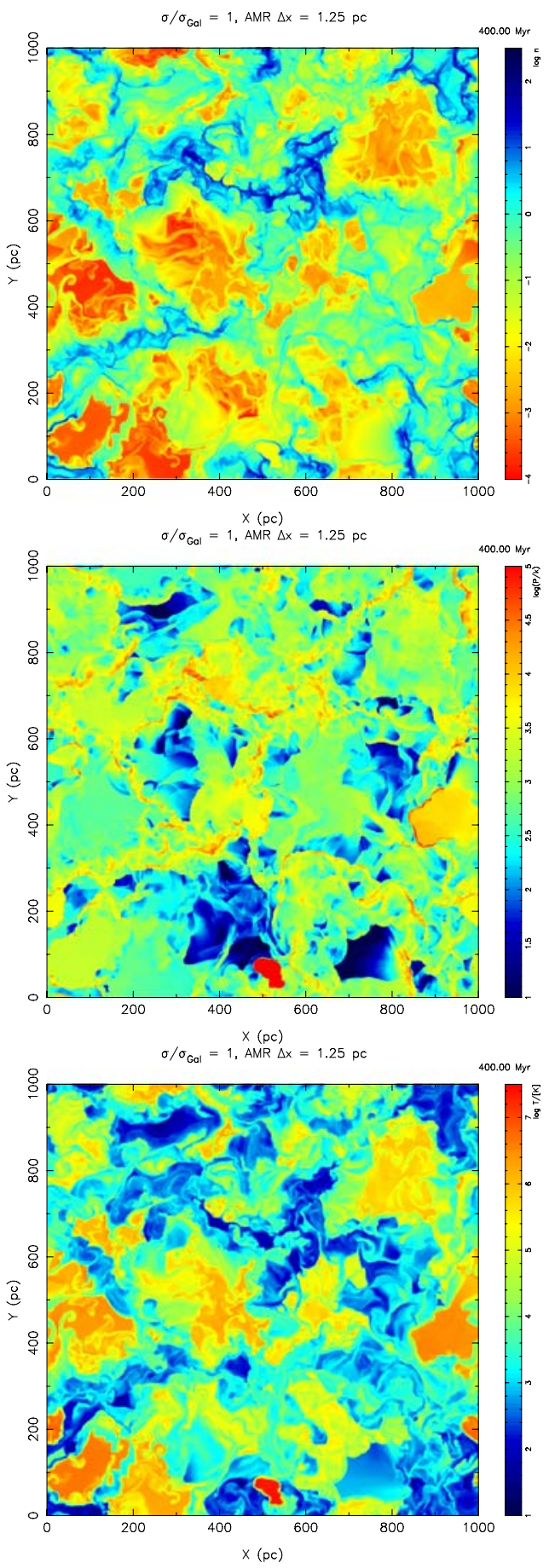
Fig. 2 Top: Time evolution of the volume filling factors of the cold $\left(T \leq 10^{3} \mathrm{~K}\right.$, black curve), cool $\left(10^{3}<T \leq 10^{4} \mathrm{~K}\right.$, blue curve), warm

$\left(10^{4}<T \leq 10^{5.5} \mathrm{~K}\right.$, green curve $)$, and hot ( $T>10^{5.5} \mathrm{~K}$, red curve) gas phases for a Galactic supernova rate $\sigma / \sigma_{G a l}=1$. The finest AMR level resolution is 1.25 pc. Bottom: Comparison of the average pressures $\left\langle P_{\text {ram }}\right\rangle$ (ram pressure, green), $\left\langle P_{\mathrm{th}}\right\rangle$ (thermal pressure, black) and $\left\langle P_{\text {mag }}\right\rangle$ (magnetic pressure, $r e d$ ) as a function of temperature in the Galactic disk $(|z| \leq 250 \mathrm{pc})$ averaged over temperature bins of $\Delta \log T=0.1 \mathrm{~K}$
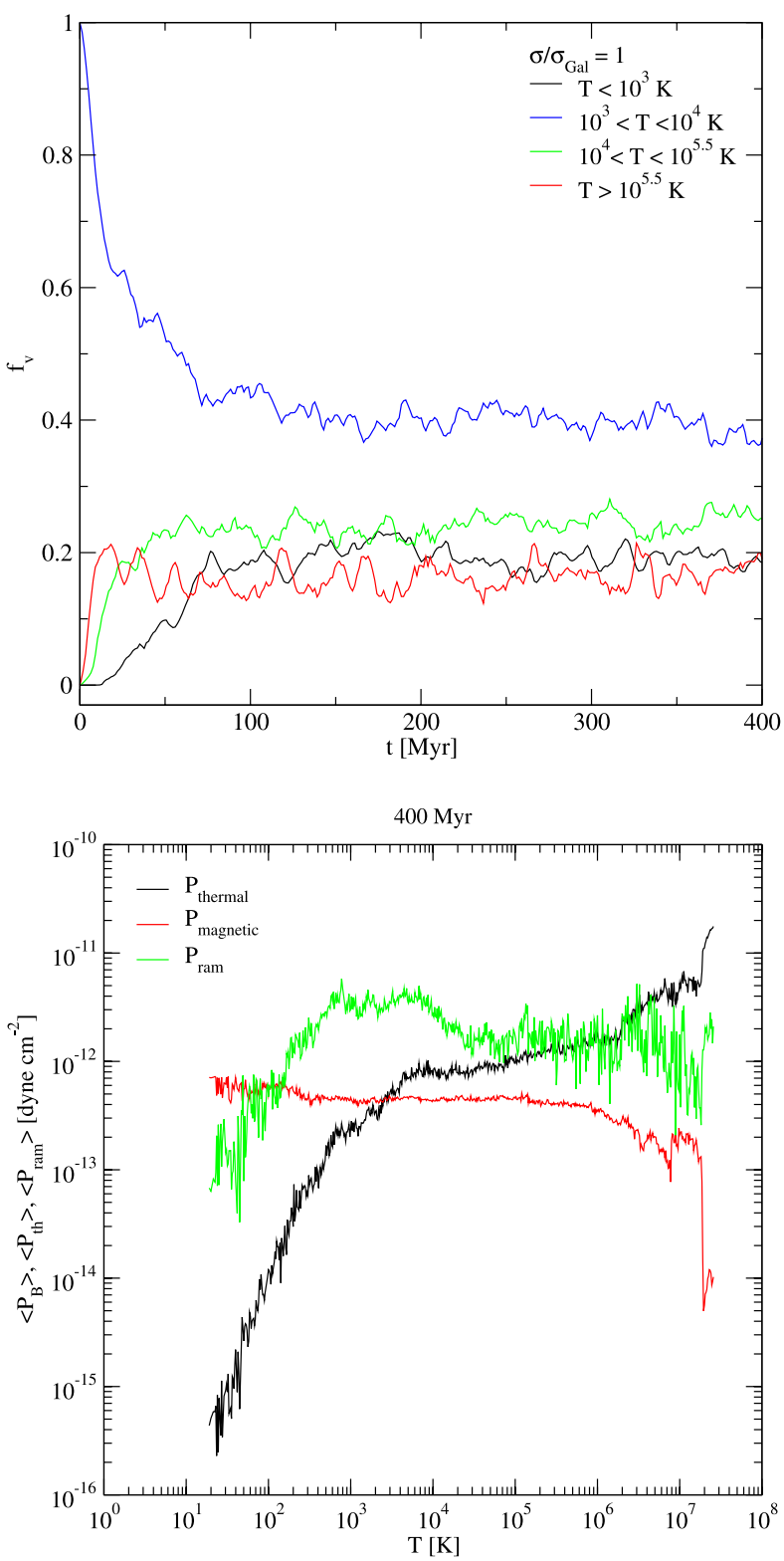

In Fig. 3 (top panel) we show the magnetic field structure perpendicular to the Galactic plane, which is characterized by a tangled topology, with a significant vertical field component, as well as showing loops and bubbles expanding away from the disk. The temperature field in the midplane (Fig. 3, bottom panel), clearly visualizes the elongated bubble structures due to magnetic tension forces of an initially disk parallel field geometry (for details see de Avillez and Breitschwerdt 2005a).

In order to proceed modeling the Local Bubble, we will now use these global ISM simulations as a typical background medium, in which a SN driven bubble evolves. Therefore 

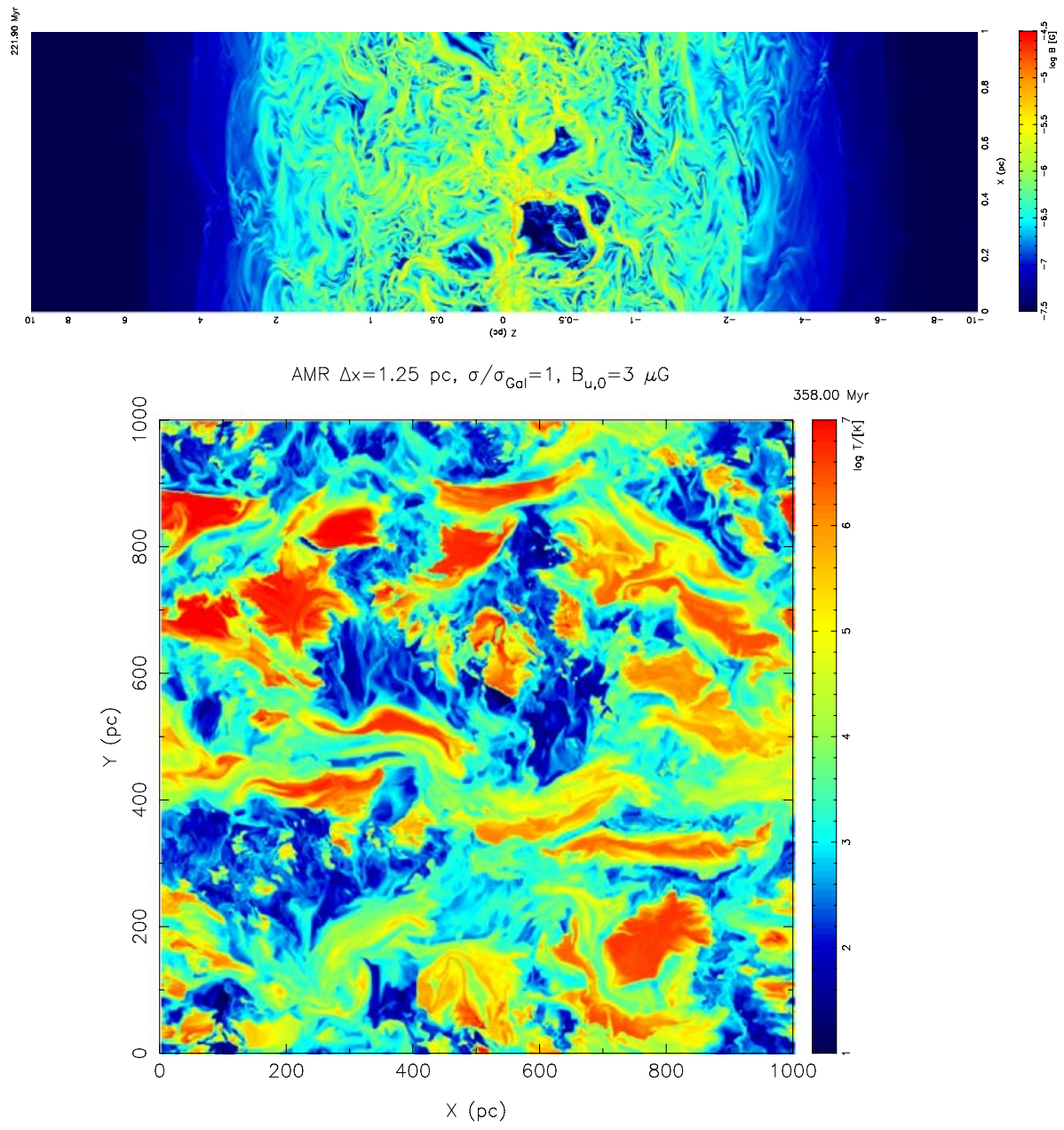

Fig. $32 \mathrm{D}$ slices of a magnetized ISM as inferred from 3D AMR MHD simulations (cf. de Avillez and Breitschwerdt 2005a). The colour coding refers to red equals high temperature/high magnetic field, and blue low temperature/low field. Top: Magnetic field distribution perpendicular to the Galactic disk at an evolution time of $t=221.9 \mathrm{Myr}$. The average initial field in the galactic plane was $B=3.1 \mu \mathrm{G}$ and parallel to the disk. The scale above and below $z= \pm 0.5 \mathrm{kpc}$ is shrunk in size to fit the picture. Magnetized loops far above the disk are clearly visible, as well as small scale turbulent structures. Bottom: Temperature distribution in the Galactic disk after $358 \mathrm{Myr}$ of evolution. Interstellar bubbles, visible as red blotches, are preferentially elongated due to the presence of the magnetic field

our next task will be to determine the stellar cluster which is responsible for the generation of the LB.

\subsection{Modeling the Local Bubble}

Modeling the LB has been so far a considerable challenge. The reasons are the following: firstly we are located inside, and therefore have some difficulty in determining the bubble extension observationally. Considering the soft X-ray flux, this can only be done by making some restrictive assumptions about the emission measure, e.g. by calibrating the flux in 
some direction (cf. Snowden et al. 1998). In contrast, the extension of the local Hi cavity seems to be known to higher precision, since NaI absorption line studies towards more than 1000 background stars (Lallement et al. 2003) yield a better spatial resolution, at least at low galactic latitudes. Secondly, we have no information about an exciting star cluster, and hence lack the most important initial condition: the energy input rate as a function of time. This is in noteworthy contrast to the neighbouring Loop I superbubble, which still harbours young massive stars that feed energy into the bubble by winds and SN explosions. The LB however seems to be extinct. Therefore in the past modeling its evolution has been based on leaving the $\mathrm{SN}$ rate as a free parameter, and assuming some ambient density distribution to begin with. Smith and Cox (2001) have argued that 3 SN explosions, with the last one occurring about 5 Myr ago would be sufficient to reproduce the soft X-ray data. However, as we shall see below, the assumption of a constant ambient density is already critical for an evolved superbubble, because the older and the larger the bubble becomes, the more its shape and expansion will be controlled by the ambient density and pressure gradients. Thus initially, the bubble will be roughly spherical, but later on will become more and more asymmetric according to the inhomogeneity of the surrounding medium. We have therefore ventured to provide a "realistic" background medium, by evolving a SN driven ISM (see Sect. 2.2) for $200 \mathrm{Myr}$ and then let the bubble expand into such a pre-structured inhomogeneous medium.

We have solved the problem of the missing OB star cluster, responsible for the creation of the LB, in the following way. We were searching the Hipparcos data base for moving groups with the idea that stars have traveled in the past through the region now occupied by the LB. In a first analysis (Berghöfer and Breitschwerdt 2002), we used the data provided by Asiain et al. (1999a, 1999b) who searched for moving groups in the solar neighbourhood and identified the Pleiades subgroup B1 as one of its youngest members. We then calculated the trajectory of this group backwards in time and found that it crossed the LB region, entering it about $15 \mathrm{Myr}$ ago and leaving it just about $1 \mathrm{Myr}$ from now. The remaining 27 stars of B1 are of spectral type A or later, and, if their masses are fitted to an IMF (e.g., Massey et al. 1995), yields the cumulative mass of stars more massive than 8 solar masses. If we bin this, somewhat arbitrarily, into stars with integer solar masses with bin size of 1 solar mass, we obtain $21 \mathrm{SN}$ candidates, two of which are now in the neighbouring Loop I bubble. Hence we concluded that $19 \mathrm{SN}$ explosions must have occurred, with the most massive star having had 20 solar masses. The main sequence life times were calculated using a formula by Stothers (1972). This analysis, although possessing some ad hoc elements, reveals a conclusive picture of the origin of the LB. The fact that Loop I is co-evolving is in our view rather a support than a counter argument for this model. Taking into account the existence of other nearby bubbles, like e.g. the Eridanus superbubble, seems to be consistent with the idea that the nearby ISM is a region of active star formation. Nevertheless, we tried to remove the assumption that the LB was the result of a particular moving group star cluster, and searched $\mathrm{ab}$ initio the whole region accessible to Hipparcos (supplemented by radial velocity data of the ARIVEL catalogue), which turned out to be a volume of $400 \mathrm{pc}$ in diameter centered on the solar circle. This analysis is described in detail by Fuchs et al. (2006, 2008). It proved that our idea of a moving group was basically correct with no other groups or interlopers being present. Hence the SN explosions inferred from the Pleiades subgroup B1 and the ones inferred from the analysis of Fuchs et al. (2006) must have been the same (see also Fuchs et al. 2008).

ROSAT PSPC observations have shown (Egger and Aschenbach 1995) that soft X-rays are absorbed by a nearby neutral shell, which is most likely the result of an interaction between the LB and the Loop I superbubble. Therefore the expansion of the LB has to be simulated along with that of Loop I, which is driven by explosions of the Sco Cen association 
members. Again, using the stellar content from observations and applying an IMF, we were able to follow the expansion of the Loop I bubble in space and time. The explosion site was chosen such as to give the correct distance from the solar circle.

The simulations were carried out by using the 3D parallelized adaptive mesh refinement (AMR) hydrocode EVAF (Évora-Vienna Astrophysical Fluid) code to track small scale structures down to $1.25 \mathrm{pc}$, where necessary, and to follow the LB and Loop I evolution in an ISM, driven by SNe types Ia, Ib+c and II (with canonical explosion energies of $10^{51} \mathrm{erg}$ ) occurring at the Galactic rate (taken from Cappellaro et al. 1999), on a Cartesian grid of $0 \leq(x, y) \leq 1 \mathrm{kpc}$ size in the Galactic plane, and $-10 \leq z \leq 10 \mathrm{kpc}$ perpendicular to it (see also Sect. 2.1).

\subsection{Results on the Local Bubble}

In order to provide as a realistic as possible background medium for the LB to expand into, we chose an ISM simulation, for which global dynamical equilibrium has been established, i.e. after $200 \mathrm{Myr}$ of Galactic evolution time. Moreover, we ensured that a sufficient amount of cold gas, to be consumed by star formation, was present in the region where we placed the origin of the LB (and Loop I as well). Indeed, the Sco Cen cluster, was put (arbitrarily) at $(375,400)$ pc (cf. Fig. 4). We then followed the trajectory of the Pleiades moving group B1, whose SNe in the LB went off along a path crossing the location at $(175,400)$ pc, chosen to match the present observed distances from the Sun and the Sco Cen. Due to small peculiar motions of the local gas, the LB will be tied to the local standard of rest (LSR; for details of the moving group kinematics see Berghöfer and Breitschwerdt, 2002). Periodic boundary conditions are applied along the four vertical boundary faces, while outflow boundary conditions are imposed at the top $(z=10 \mathrm{kpc})$ and bottom $(z=-10 \mathrm{kpc})$ boundaries. The simulation time of the LB and Loop I evolution run was an additional $30 \mathrm{Myr}$, which is somewhat larger than the age of the exciting star clusters.

The SN explosions along the cluster trajectory were generating a coherent LB structure (for details see Breitschwerdt and de Avillez 2006), which was meticulously followed in time. The bubbles, which, as it happens, are generated almost coevally, are smooth and spherical in the beginning, but develop internal structure after $8 \mathrm{Myr}$ of evolution time. This is due to the inhomogeneity of the ambient ISM and becomes progressively more important with time. The present time is fixed by comparing the LB extension with observations, which amounts to $t_{\mathrm{LB}} \simeq 14-15 \mathrm{Myr}$ after the first explosion. As we shall see below, the average column density of OVI absorption lines toward background stars (inside and beyond the LB) as measured by the FUSE and Copernicus satellites can be nicely reproduced for $t_{\mathrm{LB}}=14.5 \mathrm{Myr}$. Thus the last SN explosion just occurred 0.5 Myr before the present time, and the LB is becoming extinct, while Loop I with its larger stellar content is still active (see Fig. 4 top).

Both bubbles are bounded by shells whose interaction generates Rayleigh-Taylor instabilities due to a larger pressure in Loop I with respect to the LB, in agreement with a linear stability analysis of Breitschwerdt et al. (2000). After becoming nonlinear this leads to the formation of cloudlets, which will travel towards the solar system from the Sco Cen region, in agreement with our simulations and observations of local clouds with measured velocities of about $26 \mathrm{~km} / \mathrm{s}$. On the basis of our simulations we further predict that the interaction shell will break up in $3 \mathrm{Myr}$ from now, allowing mass transfer of hot gas from Loop I to the LB, and in $\sim 15 \mathrm{Myr}$ from now the bubbles will have merged (see Fig. 4 bottom).

In CIE, OvI is most abundant at $T \sim 3 \times 10^{5} \mathrm{~K}$, whereas soft X-Ray emission of a thermal plasma requires a temperature of $\sim 10^{6} \mathrm{~K}$. It turns out that between 14.5 and 14.7 Myr, 
Fig. 4 Top: Colour-coded temperature map in the range $10 \leq T \leq 10^{7} \mathrm{~K}$ for a slice through the data cube (representing the Galactic midplane) of a 3D LB high-resolution simulation representing the present time (i.e. 14.5 Myr after the first explosion) with the LB centred at (175, 400) pc and Loop I shifted $200 \mathrm{pc}$ to the right. Bottom: Same, showing the "future" of the LB and Loop I at $t=29.7$ Myr. Note that part of the LB has merged with the ISM, part has been engulfed by Loop I (for details, see Breitschwerdt and de Avillez 2006)

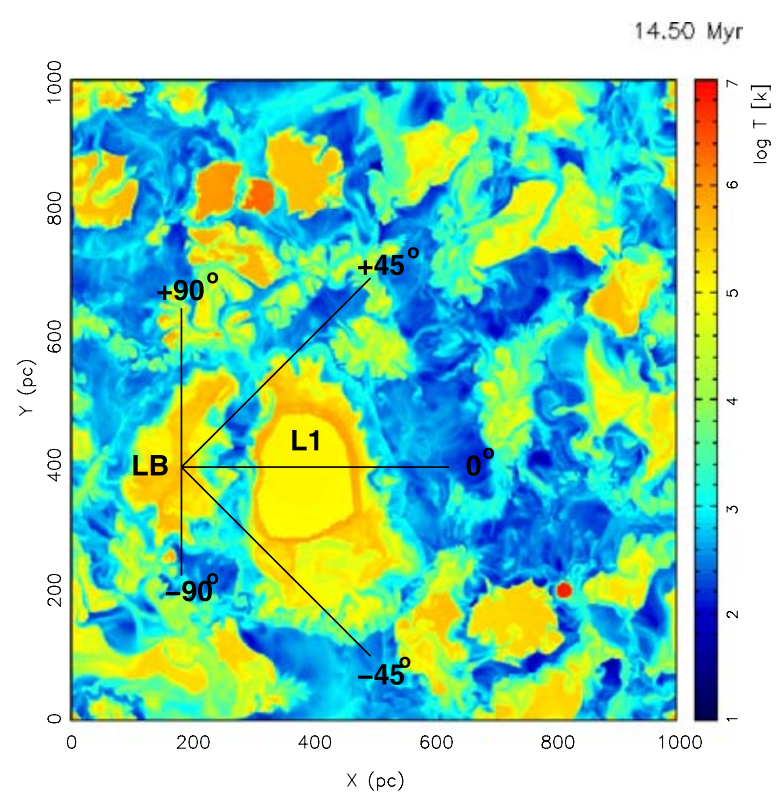

29.70 Myr

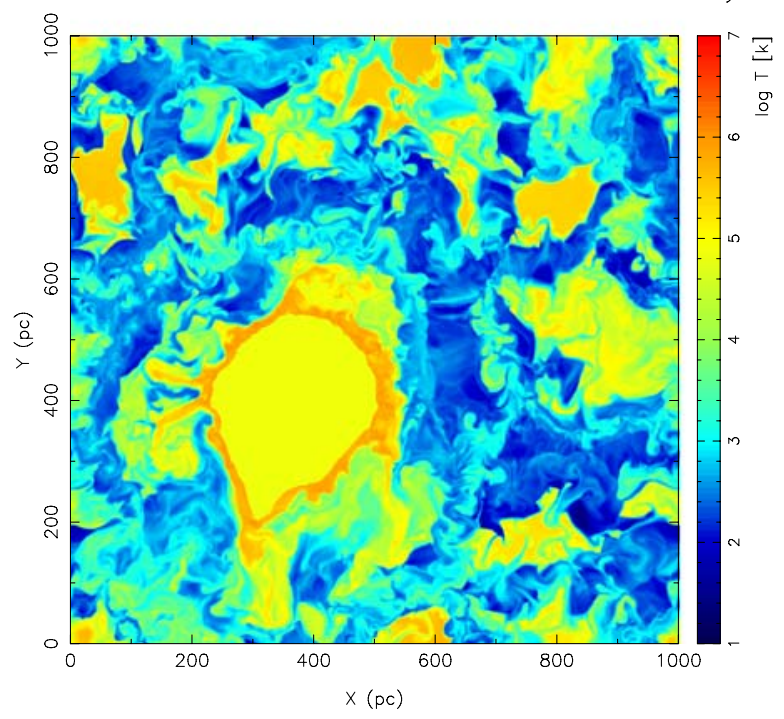

cooler (OvI absorbing) and hotter (X-ray emitting) gas co-exist. This is a direct result of the turbulent and inhomogeneous density and temperature structure in realistically evolved superbubbles. Turbulent mixing generates gas in the whole thermally unstable regime between $10^{5}-10^{6} \mathrm{~K}$. While the amount of OVI in the LB has been measured fairly accurately (see Sect. 3), the source of the soft X-ray emission is more elusive. First of all, it is more difficult to localize, as there are most likely no dense clouds inside the LB, which can be used for shadowing experiments, thus allowing us to separate back- and foreground emission. Secondly, an as yet uncertain amount of soft X-rays will be produced locally by solar wind 
charge exchange reactions with heliospheric plasma (Cravens 2000; see also Koutroumpa 2008; Shelton 2008). And thirdly, the LB plasma need not be in CIE. It has been shown that if a plasma undergoes fast adiabatic expansion its kinetic temperature will drop, but recombination can not follow fast enough, thus mimicking an "overionised" plasma (similar to the solar wind), where recombination is delayed. In essence, the gas could be at a few times $10^{5} \mathrm{~K}$, but still emit copiously in soft X-rays (Breitschwerdt and Schmutzler 1994). We are currently investigating this possibility.

\section{Comparison with Observations}

We have determined the amount of OVI along the lines of sight (LOS) for an ISM plasma in CIE with solar abundances (Anders and Grevesse 1989; for details see de Avillez and Breitschwerdt 2005b). To measure the OVI column density distribution inside LB and Loop I, we took 91 LOS extending from the Sun and crossing Loop I (the hot pressured region 200 pc to the right of the LB) from an angle of $-45^{\circ}$ to $+45^{\circ}$ as marked in the top panel of Fig. 4. Figure 5 (top) shows the history (for $14.3 \leq t \leq 14.7 \mathrm{Myr}$ ) of the variation of the LOS averaged OVI column densities, $\langle\mathrm{N}(\mathrm{OVI})\rangle$, with distance along these LOS. They decrease steeply with time after the last SN in the LB has exploded, from $4 \times 10^{13}$ to $3 \times 10^{11}$ $\mathrm{cm}^{-2}$, because no further heating is taking place. For LOS sampling gas from outside the LB (i.e., $l_{L O S}>100 \mathrm{pc}$ ) we have $\langle\mathrm{N}(\mathrm{OVI})\rangle>2 \times 10^{13} \mathrm{~cm}^{-2}$. The histograms of column densities obtained in the $91 \mathrm{LOS}$ for $t=14.5$ and 14.6 Myr (Fig. 5, bottom) show that for $t=14.6 \mathrm{Myr}$ all the LOS have column densities smaller than $10^{12.9} \mathrm{~cm}^{-2}$, while for $t=14.5$ Myr $67 \%$ of the lines have column densities smaller than $10^{13} \mathrm{~cm}^{-2}$ and about $50 \%$ of the

Fig. 5 Top: Averaged OVI column densities (over 91 LOS between $\pm 45^{\circ}$ as marked in Fig. 4 top) as a function of LOS path length at $14.3 \leq t \leq 14.7$ Myr of Local and Loop I bubbles evolution. Bottom: Histogram of the percentage of LOS as a function of N(OvI) within the LB at $t=14.5$ and $14.6 \mathrm{Myr}$
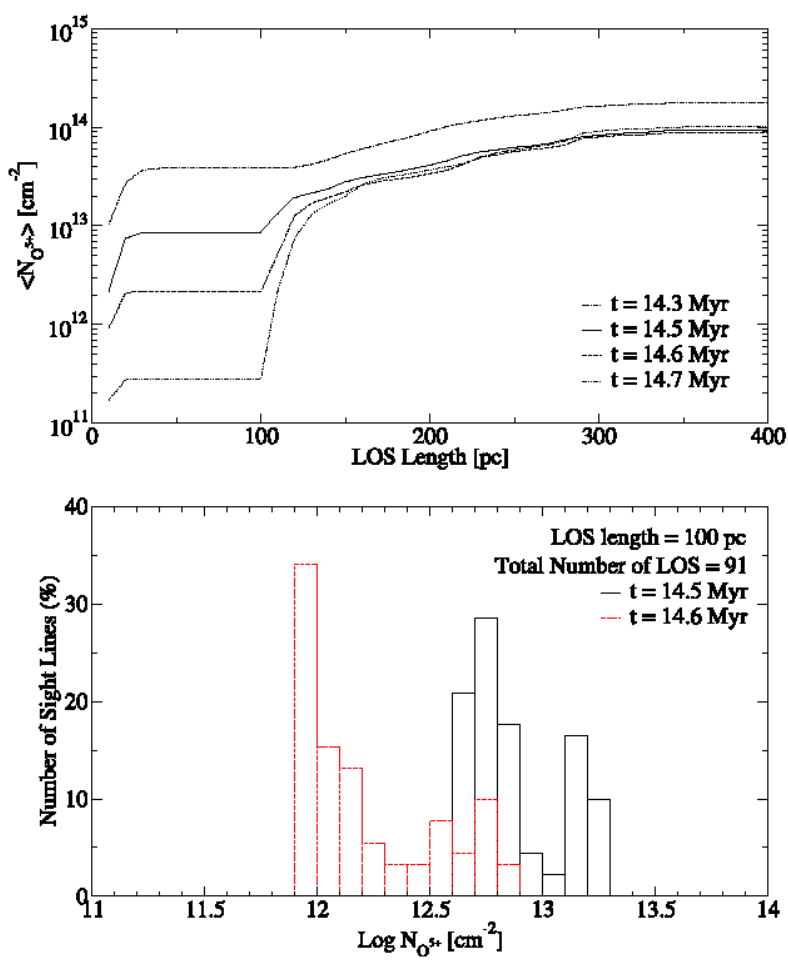
Fig. 6 Comparison between FUSE (triangles: Oegerle et al. 2005; circles: Savage and Lehner 2006) Ovi column densities with the calculated minimum, maximum and average column densities along the same 91 lines of sight (at $t=14.5 \mathrm{Myr}$ ) pointing towards Loop I as shown in Fig. 1 (top). Negative values on the ordinate derive from essentially zero equivalent absorption line widths, i.e. non-detections, due to the noise in the spectrum (cf. Oegerle et al. 2005)

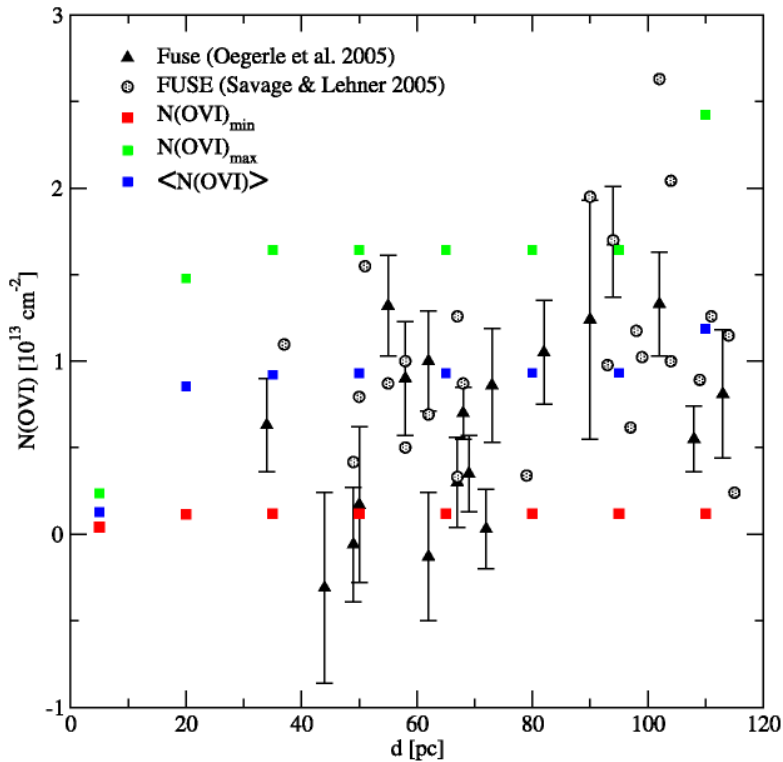

lines have $\mathrm{N}(\mathrm{OVI}) \leq 7.9 \times 10^{12} \mathrm{~cm}^{-2}$. The maximum average column density is $1.78 \times 10^{13}$ $\mathrm{cm}^{-2}$ in excellent agreement with the observations discussed in Savage and Lehner (2006), which is 1.1 times the average value of $1.6 \times 10^{13} \mathrm{~cm}^{-2}$ inferred by Shelton and Cox (1994) from a reanalysis of COPERNICUS absorption line data (cf. Jenkins 1978).

In Fig. 6 we compare FUSE data by Oegerle et al. (2005; triangles) and Savage and Lehner (2006; circles) with simulated minimum (red squares), maximum (green squares) and averaged (blue squares) column density of Ovi measurements along the 91 LOS outlined in Fig. 4 (top). It can be clearly seen in Fig. 6 that for $t=14.5 \mathrm{Myr}$ the calculated $\mathrm{N}(\mathrm{OVI})$ distribution in the LB is similar to that observed with FUSE. These results allow us to constrain the age of the LB to be $14.5 \pm_{0.4}^{0.7} \mathrm{Myr},{ }^{1}$ and capture at the same time all the relevant LB properties, such as size, age, dynamical instabilities in the interaction shell, amongst others.

Successive blast waves advance very fast through the low-density cavity, but slow down considerably later, as they run into the dense shell. As a consequence an asymmetric reverse shock leads to shear flow and turbulence inside the cavity, with a largest eddy size of a fraction of the bubble diameter, typically $l \leq 75 \mathrm{pc}$ (de Avillez and Breitschwerdt 2007). Thus the turnover time scale is about $\tau_{m} \sim l / c_{s} \leq 3.7 \times 10^{5} \mathrm{yr}$, for an averaged sound speed of $c_{s} \approx 200 \mathrm{~km} / \mathrm{s}$ after a SN explosion. As the last SN occurred about $0.5 \mathrm{Myr}$ ago, we expect that $\mathrm{SN}$ ejected oxygen inside the $\mathrm{LB}$ at present time should have a rather uniform $\mathrm{O} / \mathrm{H}$ distribution, a result that is also confirmed by FUSE observations of the $\mathrm{O} / \mathrm{H}$ ratio (Moos et al. 2002).

\footnotetext{
${ }^{1}$ We note that some uncertainty in the LB age is due to the fact that the OvI column density can be matched both during temperature increase ("ionization phase") as well as during temperature decrease ("recomnination phase") after the last explosion (see de Avillez et al. 2008).
} 


\section{Summary and Conclusions}

The solar system seems to be embedded in a region of ongoing star formation, which is testified by the existence of several nearby bubbles, like the Eridanus bubble or the Gum Nebula, and possibly radio loops II and III, just to name a few. It is therefore suggestive to assume that also the Local Bubble is the result of recent supernova explosions. The fact that the trajectory of the moving group, which harboured the progenitor stars, is not going through the geometrical centre of the LB is immaterial, since the expansion always proceeds in the direction of the largest pressure gradient. The expansion of the neighbouring Loop I superbubble, which was generated almost at the same time, corroborates the argument, because its high pressure will force the LB to expand preferentially away from it, which is fully confirmed by our numerical simulations. The analysis of the stellar content of the moving group reveals that substantially more than $3 \mathrm{SNe}$ occurred during the last $15 \mathrm{Myr}$. Again, the expansion proceeds most rapidly in the direction of least resistance, i.e. perpendicular to the Galactic disk, once a disk scale has been reached. Therefore, it is not surprising that a number of at least $14 \mathrm{SNe}$ (cf. Fuchs et al. 2006) will create a local chimney in agreement with the observations of Lallement et al. (2003).

The Local Cloud and its companions occur naturally in our model as a consequence of Rayleigh-Taylor instabilities, ensuing from the interaction between the LB and Loop I. It is probably not fortuitous that its velocity vector points away from the interaction shell of the two bubbles.

High-resolution 3D hydrodynamical simulations of the LB and Loop I show that UV data from FUSE and Copernicus - both for lines of sight within and outside the LB - can be nicely reproduced. It should be emphasized that our simulations were performed before the FUSE data were available; hence there is no "tuning" involved here. It can also be concluded from these calculations that within the computational box of $1 \mathrm{kpc} \times 1 \mathrm{kpc}$ in the Galactic plane several bubble regions are present. Hence the physical processes that drive the general ISM structures are also responsible for structuring the LISM in general and the LB in particular. A key element of the physics governing the ISM is (compressible) turbulence. Turbulent diffusion is an efficient transport process and is capable of inhibiting local thermal instabilities, thus keeping a lot of interstellar gas in thermally unstable regimes. For example, about half of the warm neutral medium is between 500 and $5000 \mathrm{~K}$, in agreement with observations by Heiles and Troland (2003).

Since the Lyman-Spitzer Colloquium "The Local Bubble and Beyond" in 1997, it is fair to state that modeling the LB has become less of a guess work, and a coherent picture seems to emerge, although of course lots of details still need to be explained.

Acknowledgements DB thanks ISSI, Ruedi von Steiger and S.F. Saliba for their organizational help with the presentation of the talk.

\section{References}

E. Anders, N. Grevesse, Geochim. Cosmochim. Acta 53, 197 (1989)

R. Asiain, F. Figueras, J. Torra, B. Chen, Astron. Astrophys. 341, 427 (1999a)

R. Asiain, F. Figueras, J. Torra, Astron. Astrophys. 350, 434 (1999b)

T. Berghöfer, D. Breitschwerdt, Astron. Astrophys. 390, 299 (2002)

E.B. Berkhuijsen, A. Fletcher (2008). arXiv:0806.4316

J.N. Bregman, Astrophys. J. 236, 577 (1980)

D. Breitschwerdt, M.A. de Avillez, Astron. Astrophys. 452, L1 (2006)

D. Breitschwerdt, S. Komossa, Astrophys. Space Sci. 272, 3 (2000) 
D. Breitschwerdt, T. Schmutzler, Nature 371, 774 (1994)

D. Breitschwerdt, J.F. McKenzie, H.J. Völk, Astron. Astrophys. 245, 79 (1991)

D. Breitschwerdt, R. Egger, M.J. Freyberg, Astron. Astrophys. 361, 303 (2000)

M.A. de Avillez, Mon. Not. R. Astron. Soc. 315, 479 (2000)

M.A. de Avillez, D. Breitschwerdt, Astron. Astrophys. 425, 899 (2004)

M.A. de Avillez, D. Breitschwerdt, Astron. Astrophys. 436, 585 (2005a)

M.A. de Avillez, D. Breitschwerdt, Astrophys. J. 634, L65 (2005b)

M.A. de Avillez, D. Breitschwerdt, Astrophys. J. 665, L35 (2007)

M.A. de Avillez, et al., Space Sci. Rev. (2008, this issue)

E. Cappellaro, R. Evans, M. Turatto, Astron. Astrophys. 351, 459 (1999)

D.P. Cox, R.K. Smith, Astrophys. J. Suppl. Ser. 134, 283 (2001)

T.E. Cravens, Astrophys. J. 532, L153 (2000)

A. Dalgarno, R.A. McCray, Annu. Rev. Astron. Astrophys. 10, 375 (1972)

R. Egger, B. Aschenbach, Astron. Astrophys. 294, L25 (1995)

B.G. Elmegreen, J. Scalo, Annu. Rev. Astron. Astrophys. 42, 211 (2004)

J.E. Everett, E.G. Zweibel, R.A. Benjamin, D. McCammon et al., Astrophys. J. 674, 258 (2008)

G.B. Field, Astrophys. J. 142, 531 (1965)

B. Fuchs, D. Breitschwerdt, M.A. de Avillez, C. Dettbarn, C. Flynn, Mon. Not. R. Astron. Soc. 373, 993 (2006)

Fuchs et al., Space Sci. Rev. (2008, this issue)

C. Heiles, T.H. Troland, Astrophys. J. 586, 1067 (2003)

E.B. Jenkins, Astrophys. J. 219, 845 (1978)

F.D. Kahn, in Investigating the Universe, ed. by F.D. Kahn (Reidel, Dordrecht, 1981)

Koutroumpa, Space Sci. Rev. (2008, this issue)

K. Kuijken, G. Gilmore, Mon. Not. R. Astron. Soc. 239, 651 (1989)

R. Lallement, B.Y. Welsh, J.L. Vergely, F. Crifo, D. Sfeir, Astron. Astrophys. 411, 447 (2003)

M.-M. MacLow, R.S. Klessen, Rev. Mod. Phys. 76, 125 (2004)

P. Massey, K.E. Johnson, K. Degioia-Eastwood, Astrophys. J. 454, 151 (1995)

W.G. Mathews, J.C. Baker, Astrophys. J. 170, 241 (1971)

C.F. McKee, J.P. Ostriker, Astrophys. J. 218, 148 (1977)

H.W. Moos, K.R. Sembach, A. Vidal-Madjar et al., Astrophys. J. Suppl. Ser. 140, 3 (2002)

W.R. Oegerle, E.B. Jenkins, R.L. Shelton, D.V. Bowen, P. Chayer, Astrophys. J. 622, 377 (2005)

B.D. Savage, K.S. de Boer, Astrophys. J. 243, 460 (1981)

B.D. Savage, N. Lehner, Astrophys. J. Suppl. Ser. 162, 134 (2006)

P.M. Shapiro, G.B. Field, Astrophys. J. 205, 762 (1976)

R. Shelton, Space Sci. Rev. (2008, this issue)

R. Shelton, D.P. Cox, Astrophys. J. 434, 599 (1994)

S.L. Snowden, R. Egger, D.P. Finkbeiner, D. McCammon et al., Astrophys. J. 493, 715 (1998)

R. Stothers, Astrophys. J. 175, 431 (1972)

R.S. Sutherland, M.A. Dopita, Astrophys. J. Suppl. Ser. 88, 253 (1993)

Tüllmann, W. Pietsch, J. Rossa, D. Breitschwerdt, R.-J. Dettmar, Astron. Astrophys. 448, 43 (2006)

C.F. von Weizsäcker, Astrophys. J. 114, 165 (1951)

M.G. Wolfire, C.F. McKee, D. Hollenbach, A.G.G.M. Tielens, E.L.O. Bakes, Astrophys. J. 443, 152 (1995) 